\title{
Acute gastroesophageal intussusception in a patient with pneumomediastinum, pneumoperitoneum, and extensive subcutaneous emphysema resulting from iatrogenic colonic perforation
}

\author{
Geum Hee Hwang, Jun Ho Kim, Kyung Hee Lee, Ga Ram Kim, Yu Jin Ku, Yong Sun Jeon, Soon Gu \\ Cho \\ Department of Radiology, Inha University Hospital, Inha University School of Medicine, Incheon, Korea \\ Correspondence to: Jun Ho Kim, MD. Department of Radiology, Inha University Hospital, Inha University School of Medicine, Inhang-ro 27, Jung- \\ gu, Incheon 22332, Korea. Email: jhkim7227@gmail.com.
}

\begin{abstract}
Acute gastroesophageal intussusception is a rare condition in which the stomach invaginates into the adjacent esophagus. We report a case of acute gastroesophageal intussusception with massive pneumomediastinum, pneumoperitoneum, and subcutaneous emphysema, effectively treated with conservative management.
\end{abstract}

Keywords: Intussusception; esophagus; stomach; computed tomography (CT)

Submitted Nov 21, 2017. Accepted for publication Apr 03, 2018.

doi: $10.21037 /$ jtd.2018.04.77

View this article at: http://dx.doi.org/10.21037/jtd.2018.04.77

\section{Introduction}

Gastroesophageal intussusception is a rare disease involving invagination of the stomach into the distal esophagus. Only a few case reports have described diagnosis with the use of gastrointestinal endoscopy (1). Furthermore, acute gastroesophageal intussusception on computed tomography (CT) imaging is extremely rare condition. To the best of our knowledge, only two cases have been reported $(2,3)$. We describe a case of acute gastroesophageal intussusception secondary to iatrogenic injury of the colon.

\section{Case presentation}

A 78-year-old man with a past medical history of diabetes mellitus visited the emergency department complaining of abdominal pain. The patient had no surgical history. The patient was referred to our tertiary hospital for further evaluation and management, with suspicion of colonic perforation during endoscopy. The abdomen was diffusely tender and distended. He did not present with hemoptysis or hematemesis. He underwent routine screening colonoscopy earlier in the day. Before colonoscopy, he was in his usual state of health. Chest radiography showed massive subcutaneous emphysema, pneumomediastinum, and pneumoperitoneum, with posterior cardiac fullness and a suspicious air pocket (Figure 1). CT of the chest, abdomen, and pelvis confirmed perforation of the descending colon and revealed massive free retroperitoneal and intraperitoneal air, and massive pneumomediastinum with extensive gas dissection throughout the neck. Axial and multiplanar reconstructed images showed a bowel-inbowel appearance of intussusception (Figure 2). Emergency diagnostic laparotomy was performed. A perforation was found in descending colon. And intraoperative findings included serosal injury and adhesion at around perforation site. About $7 \mathrm{~cm}$ long segment of descending colon was removed, including perforation site. The operator finished the operation without surgical management of gastroesophageal intussusception. Also, the patient was not treated by endoscopy. The patient was admitted to the intensive care unit for close monitoring and was managed conservatively with bowel rest, intravenous fluids, and 


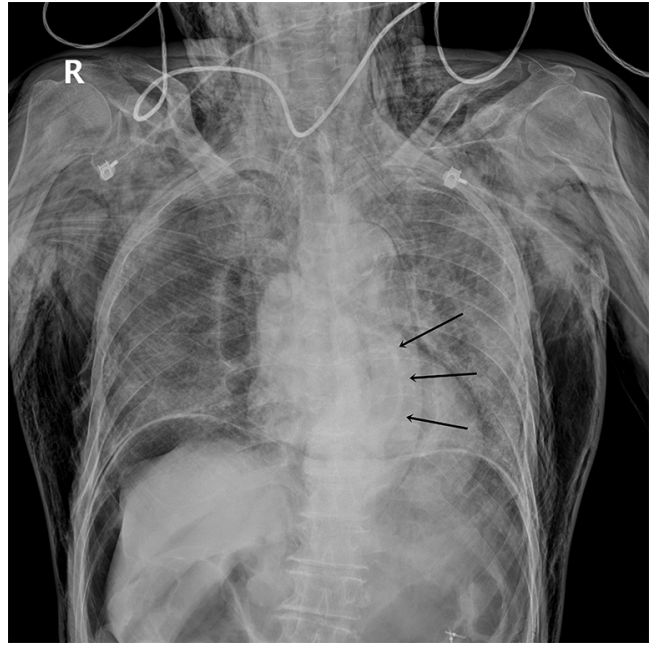

Figure 1 Chest radiography shows an air-containing tubular structure behind the cardiac shadow (arrow). There is extensive subcutaneous emphysema in the neck and chest wall, and a paracardiac gas strip. broad-spectrum antibiotics. The patient started to eat soft food at postoperative day 13. The patient's condition improved. Repeated CT of the chest, abdomen, and pelvis 20 days later showed interval improvement of the pneumomediastinum and pneumoperitoneum, in addition to resolution of the gastroesophageal intussusception (Figure 3).

\section{Discussion}

The term intussusception defines a condition in which a bowel segment (intussusceptum) folds into an adjacent segment (intussuscipiens) (4). Intussusception primarily occurs in the small bowel and colon $(2,4,5)$. Intussusception in the lower intestine is usually diagnosed with ultrasonography and CT $(4,5)$.

Gastroesophageal intussusception is an unusual condition in which the stomach is translocated into the intrathoracic esophageal lumen. Previous reports
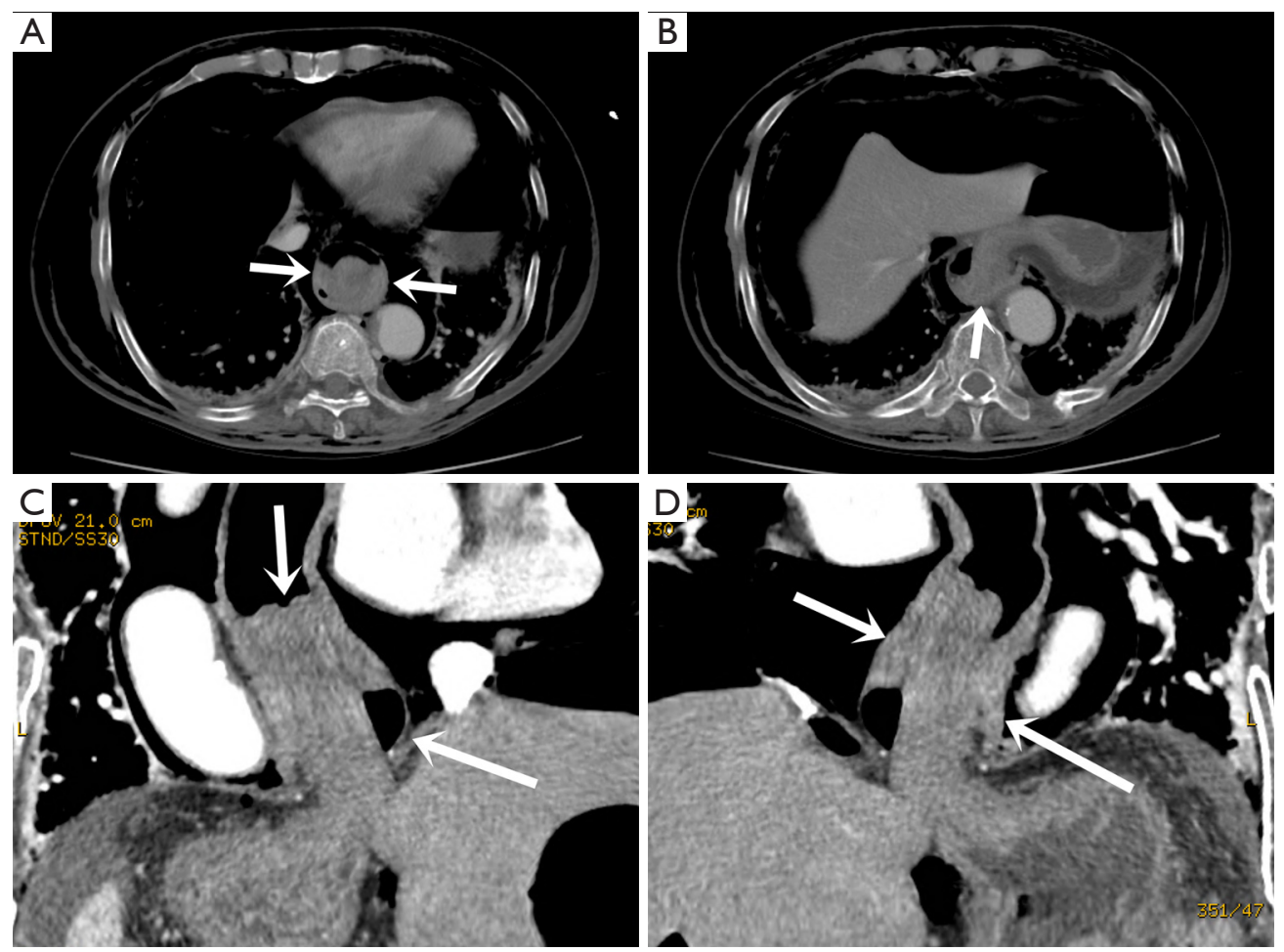

Figure 2 Axial CT shows a bowel-in-bowel configuration, in which the layers of the bowel are duplicated, forming concentric rings (arrow). (A,B) Multiplanar reconstruction images show invagination (arrow) of the stomach (intussusceptum) into the distal esophagus (intussuscipiens); (C,D) CT scan shows pneumoperitoneum and pneumomediastinum. 

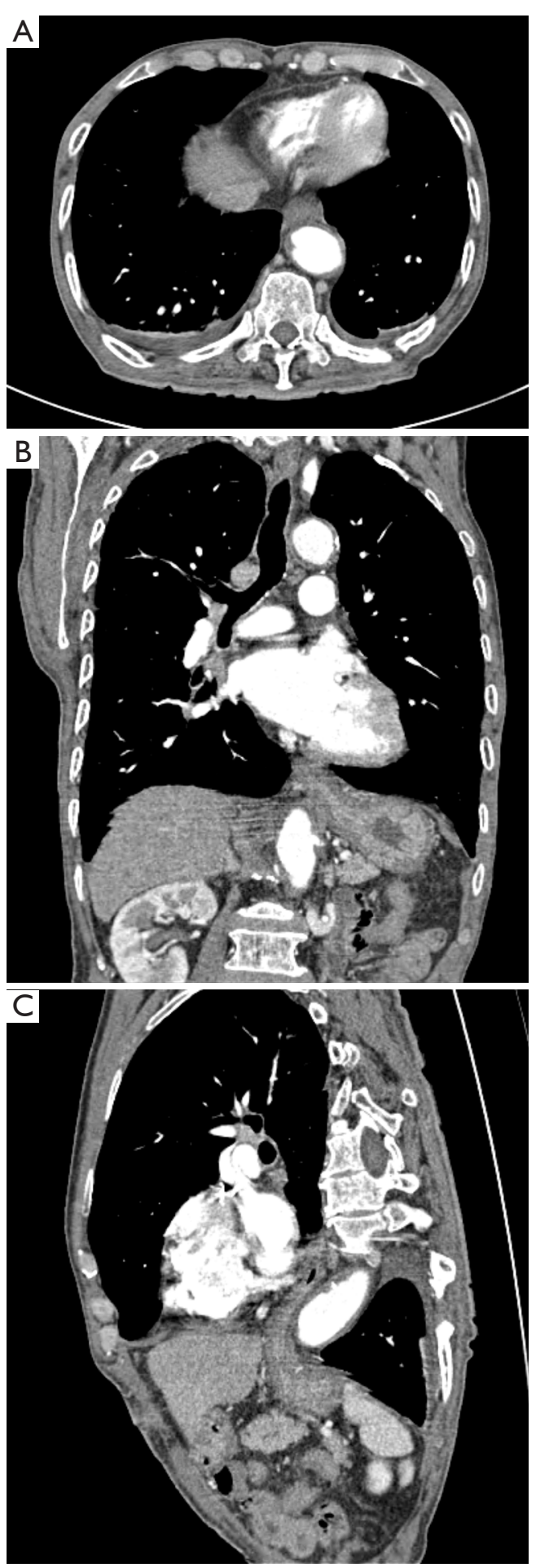

Figure 3 Follow-up axial (A) and multiplanar reconstruction $(\mathrm{B}, \mathrm{C}) \mathrm{CT}$ scan 20 days later shows complete resolution of gastroesophageal intussusception and disappearance of the pneumomediastinum, pneumoperitoneum, and subcutaneous emphysema. described cases that were diagnosed using endoscopy or fluoroscopy $(1,6)$. Gowen et al. reported large case series with gastroesophageal intussusceptions and most patients were diagnosed successfully by endoscopy (1). Only a few reported cases have been diagnosed using CT $(2,3)$. Gastroesophageal intussusception caused by achalasia has been diagnosed with CT in an adult (3), and in a child with intussusception of unclear cause (2). Intussusception was first suspected on endoscopy in the adult, and fluoroscopy in the child, and CT was performed for confirmation $(2,3)$. However, multidetector CT (MDCT) in our case clearly identified gastroesophageal intussusception. MDCT allows thinner collimation and enables the acquisition of highresolution volume data sets. Multiplanar reconstruction is readily performed and facilitates diagnosis $(5,7)$. Iterative multiplanar reconstruction images in our case showed the stomach telescoping into the distal esophagus, confirming the presence of gastroesophageal intussusception.

A previous report suggested that risk factors included eating disorders, obstruction due to congenital small intestinal lesions, sustained physical exertion, peptic disease, and pregnancy (1). In our case, CT showed extensive pneumomediastinum, pneumoperitoneum, pneumoretroperitoneum, and subcutaneous emphysema following colonoscopy with excessive air insufflation; these findings are also extremely rare (8). Previous literature suggests that retroperitoneal air might migrate through the diaphragmatic hiatus along the adventitia of great vessels to the mediastinum and pretracheal fascia to the neck because the spaces between the retroperitoneum, mediastinum, and subcutaneous tissue are anatomically continuous $(9,10)$. Intussusception in our case could have resulted from increased intraperitoneal pressure due to extensive pneumoperitoneum. The authors found no report of similar etiology in the literature.

The choice of conservative or surgical treatment for gastroesophageal intussusception remains controversial (1-3). Previous literature reported successful treatment with surgery and manual reduction $(2,3)$. Other case reports described most of patients except few cases with massive upper gastrointestinal tract hemorrhage and congenital lesions obstructing the duodenum were effectively managed with medical or conservative treatment. In the present case, emergency laparotomy was performed because the patient presented with diffuse, severe abdominal pain. As he had no symptoms, such as chest pain, vomiting, or hematemesis, conservative management was chosen. Follow-up CT revealed complete resolution of gastroesophageal 
intussusception, probably due to decreased intraperitoneal pressure after surgical management.

In conclusion, to our knowledge, this case report is the first to describe gastroesophageal intussusception with pneumomediastinum, pneumoperitoneum, and extensive subcutaneous emphysema resulting from iatrogenic colonic perforation. MDCT was useful in diagnosis.

\section{Acknowledgements}

This work was supported by an Inha University Research Grant (2018).

\section{Footnote}

Conflicts of Interest: The authors have no conflicts of interest to declare.

Ethical Statement: This case study was approved by Institutional Review Board (INHAUH 2017-10-011) for Inha University Hospital and written informed consent was obtained from the patient for publication of this manuscript and any accompanying images.

\section{References}

1. Gowen GF, Stoldt HS, Rosato FE. Five risk factors identify patients with gastroesophageal intussusception. Arch Surg 1999;134:1394-7.

2. Lukish JR, Eichelberger MR, Henry L, et al. Gastroesophageal intussusception: a new cause of acute esophageal obstruction in children. J Pediatr Surg

Cite this article as: Hwang GH, Kim JH, Lee KH, Kim GR, Ku YJ, Jeon YS, Cho SG. Acute gastroesophageal intussusception in a patient with pneumomediastinum, pneumoperitoneum, and extensive subcutaneous emphysema resulting from iatrogenic colonic perforation. J Thorac Dis 2018;10(5):E347-E350. doi: 10.21037/jtd.2018.04.77
2004;39:1125-7.

3. Zhang J, Guan Z, Zhang P. Oesophagogastric invagination. Ann R Coll Surg Engl 2017;99:e202-3.

4. Park NH, Park SI, Park CS, et al. Ultrasonographic findings of small bowel intussusception, focusing on differentiation from ileocolic intussusception. Br J Radiol 2007;80:798-802.

5. Floemer F, Bissig H, Oertli D, et al. Multislice CT in adult colocolic intussusception: case report and review of the literature. Emerg Radiol 2008;15:361-6.

6. Rudnick JP, Ferrucci JT Jr, Eaton SB Jr, et al. Esophageal pseudotomor: retrograde prolapse of gastric mucosa into the esophagus. Am J Roentgenol Radium Ther Nucl Med 1972;115:253-6.

7. Choi HJ, Lee KH, Kim NH, et al. The usefulness of combined axial and coronal computed tomography for the evaluation of metastatic supraclavicular lymph nodes. Clin Imaging 2015;39:608-12.

8. Yang J, Liu WQ, Dong J, et al. Pneumothorax, pneumomediastinum, pneumoperitoneum and extensive subcutaneous emphysema resulting from endoscopic mucosal resection secondary to colonoscopy: A case report. Oncol Lett 2016;11:2763-7.

9. Zhang JL, Wang J, Li RQ, et al. Subcutaneous emphysema and buccopharyngeal submucosal emphysema after retroperitoneal laparoscopic surgery and upper airway obstruction. Br J Anaesth 2013;110:317-8.

10. Siddiqui UT, Shahzad H, Raja AJ. Pneumoperitoneum, pneumoretroperitoneum, pneumomediastinum and extensive subcutaneous emphysema in a patient with ulcerative colitis: A case report. Int J Surg Case Rep 2015;17:12-5. 